\title{
Measurement and analysis of two- and three-particle correlations
}

\author{
M. Csanád ${ }^{a}$ for the PHENIX* Collaboration

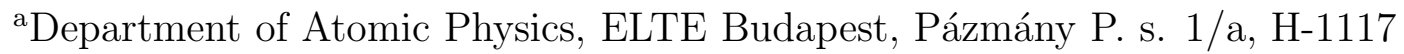

Allowed regions of core fraction versus partial coherence are obtained from analysis of both two- and three-pion Bose-Einstein correlations measured at PHENIX in $\sqrt{s_{\mathrm{NN}}}=200$ $\mathrm{GeV} \mathrm{Au}+\mathrm{Au}$ collisions. Two-pion Bose-Einstein correlation functions for different average transverse momenta are used to analyze chiral symmetry restoration.

\section{Introduction}

Correlation functions are important to see the collective properties of particles and space-time structure of the emitting source, eg. the observed size of a system can be measured by two particle Bose-Einstein correlations [1]. The $m_{t}$ dependent strength of two-pion correlations provides information about $\mathrm{U}_{\mathrm{A}}(1)$ symmetry restoration in the source [ 2, 3, 4, 5]. From a consistent analysis of two- and three-particle correlations one can establish an experimental measure of thermalization and coherence in the source [ 6 .

\section{Data Analysis}

We studied $70 \mathrm{M} \sqrt{s_{\mathrm{NN}}}=200 \mathrm{GeV}$ RHIC Au+Au collisions recorded at PHENIX during Run 4. Charged tracks were detected by the Drift Chamber and Pad Chambers. The Time Of Flight detector and the Electromagnetic Calorimeters were used to identify $\pi^{+}$ particles, and we had $200 \mathrm{M} \pi^{+}$'s, $900 \mathrm{M} \pi^{+}$pairs and more than $4 \mathrm{G} \pi^{+}$triplets in this analysis. We measured from this data sample the two-pion correlation function $C_{2}$ as a function of $q_{\text {inv }}=\sqrt{\left(k_{1}-k_{2}\right)^{2}}$ and the three-pion correlation function $C_{3}$ as a function of $q_{\text {inv }}$ of the three pairs in the triplet, $q_{12}, q_{23}, q_{31}$.

Our method of Coulomb-correction is based on the solution of the two-body Coulombproblem. A symmetrized asymptotically correct three-body Coulomb wave-function is built up from the two-body solution. The source function is then integrated with the absolute square of this three-body Coulomb-wave function as the density function, and the same is done for a symmetrized three-body plain-wave function. The ratio of the two gives the Coulomb-correction factor [ 7]. For the final Coulomb-correction we utilized a core/halo type of picture of the source and iterated $\lambda$ and $R$ in a self-consistent manner until convergence.

The fits were done using three different shapes, Gauss, Levy and Edgeworth, described in ref. [8]. We fitted only those points with $q_{\text {inv }}>0.02 \mathrm{GeV} / c$ due to a non-BEC and non-Coulomb structure seen at low $q_{\text {inv }}$ (see the gray lines on fig. 11).

\footnotetext{
*For the full list of PHENIX authors and acknowledgments, see appendix 'Collaborations' of this volume.
} 

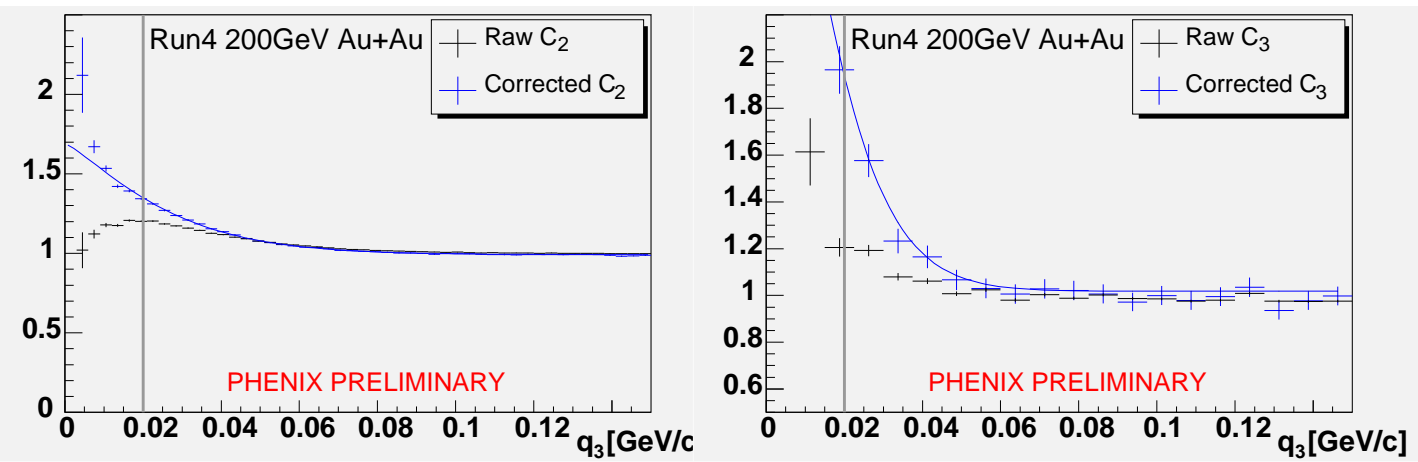

Figure 1. Raw- and Coulomb-corrected correlation functions for $0.2<p_{t}<2.0 \mathrm{GeV} / c$ and $0-92 \%$ centrality with Levy fits. Points with $q_{\text {inv }}<0.02 \mathrm{GeV} / c$ were excluded from the fits.

\section{Results}

First, we fitted two- and three-particle correlation functions for $0.2<p_{t}<2.0 \mathrm{GeV} / c$ and $0-92 \%$ centrality. Here only the Levy fits had acceptable confidence levels. These and the Coulomb-corrected correlation functions are shown on fig. 11. The three-dimensional $C_{3}$ was projected onto the $q_{12}=q_{23}=q_{31}$ line.

Let us consider the ratios $f_{c}=N_{c}(p) / N_{1}(p)$ (fractional core) and $p_{c}=N_{c}^{p}(p) / N_{c}(p)$ (partial coherence), and take the equations (see eqs. 57 and 58 in ref. [ [8]):

$$
\begin{aligned}
C_{2}\left(p_{1} \simeq p_{2}\right) & =1+f_{c}^{2}\left[1-p_{c}^{2}\right] \\
C_{3}\left(p_{1} \simeq p_{2} \simeq p_{3}\right) & =1+3 f_{c}^{2}\left[1-p_{c}^{2}\right]+2 f_{c}^{3}\left[\left(1-p_{c}\right)^{3}+3 p_{c}\left(1-p_{c}\right)^{2}\right]
\end{aligned}
$$

From this we calculated the allowed $f_{c}$ vs $p_{c}$ region, using the $3 \sigma$ contours from eqs. 1 2. see fig. 2. The size of the region obtained from $C_{3}$ is much larger due to the smaller number of triplets compared to pairs.

Compared to former NA44 analysis (see refs. [ 6, 9, 10]), the resulting allowed region is at a little bit higher $f_{c}$ values, because our $f_{c}$ was obtained from Levy fits and not from Gauss, as confidence level of latter was unacceptable.

In the second part of the analysis, we have analyzed two-pion correlation functions in $10 p_{t}$ bins from $0.2 \mathrm{GeV} / c$ to $0.5 \mathrm{GeV} / c$ with $0-92 \%$ centrality, using the invariant momentum difference $q \equiv q_{\text {inv }}$ as a variable and the following shapes (in $\hbar c=1$ units):

$$
\begin{aligned}
\text { Gauss: } & C_{2}(q)=1+\lambda \exp \left(-|R q|^{2}\right) \\
\text { Levy: } & C_{2}(q)=1+\lambda \exp \left(-|R|^{\alpha}\right) \\
\text { Edgeworth: } & C_{2}(q)=1+\lambda \exp \left(-|R q|^{2}\right)\left(1+\frac{\kappa_{3}}{3 !} H_{3}(\sqrt{2}|R q|),\right.
\end{aligned}
$$

where $H_{3}$ is the third Hermite polynomial, and $\kappa_{3}$ it's coefficient (the Edgeworth form is a model-independent expansion, of which we keep only the first term, to measure deviation from the Gauss form). See more details in ref. [11.

The parameters as a function of average transverse mass $\left(\lambda\left(m_{t}\right), R\left(m_{t}\right), \alpha\left(m_{t}\right)\right.$ and $\left.\kappa_{3}\left(m_{t}\right)\right)$ for Gauss, Levy and Edgeworth fits are shown on fig. 3. The $\alpha$ parameter of the 


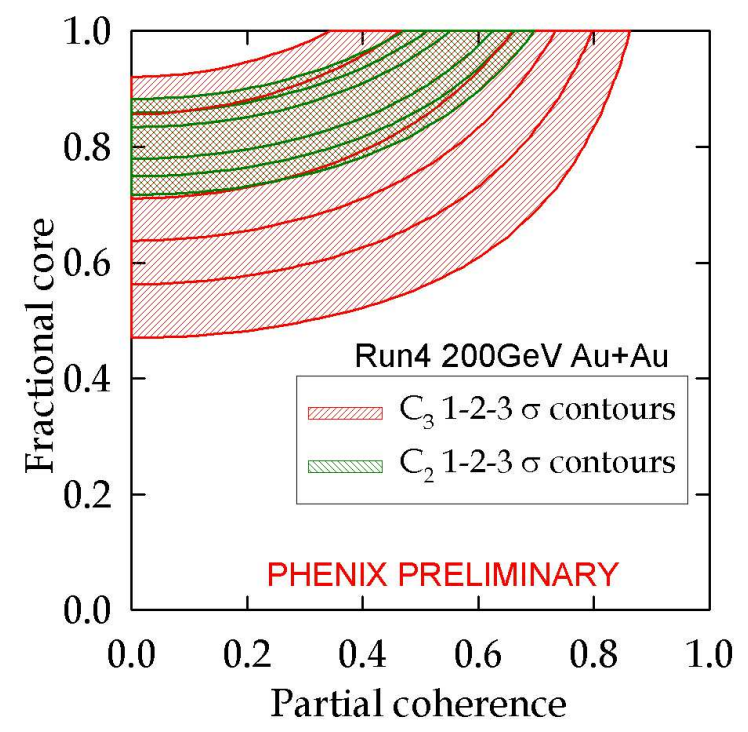

Figure 2. Allowed region of partial coherence versus fractional core extracted from Levy fits. Contours obtained from $C_{3}$ are much larger due to smaller statistics of $\pi^{+}$triplets compared to $\pi^{+}$pairs.

Levy distribution controls the long-range source in a similar way as the $\lambda$ parameter, so fitting both makes the fit underconstrained. Hence we did fits also with fixed $\alpha$, and for comparison, also with fixed $\kappa_{3}$. These latter fits show the same $\lambda\left(m_{t}\right)$ behavior as in the Gaussian case.

The $m_{t}$ dependence of $\lambda$ can be used to extract information on the mass-reduction of the $\eta^{\prime}$ meson, a signal of the $\mathrm{U}_{\mathrm{A}}(1)$ symmetry restoration in hot and dense matter (see ref. [2]). A comparison of the measurements of fig. 3] with model calculations of ref. [ [2] using Fritiof results for the composition of the long-lived resonances and a variation of the $\eta^{\prime}$ mass is presented in fig. 4 .

\section{Conclusion}

From simultaneous analysis of two- and three-pion correlations we obtained an allowed region of partial coherence versus core fraction. Nonzero partial coherence is allowed when having larger core size, at total incoherence $\left(p_{c}=0\right)$ the core fraction is slightly higher than previous analysis using Gaussian fits (see refs. [6, 9, 10, 12]). This is due to the fact that we used Levy fits, the only investigated one that gave acceptable confidence levels.

We also analyzed the $m_{t}$ dependence of the $\lambda$ parameter obtained from various fits. Gauss fit results agree with former PHENIX measurements (see ref. [12]). Regarding $\mathrm{U}_{A}(1)$ symmetry restoration, we conclude that at present, results are critically dependent on our understanding of statistical and systematic errors, and additional analysis is required to make a definitive statement.

\section{REFERENCES}

1. R. Hanbury Brown and R. Q. Twiss, Nature 178, 1046 (1956)

2. S. E. Vance, T. Csörgö and D. Kharzeev, Phys. Rev. Lett. 81, 2205 (1998) 


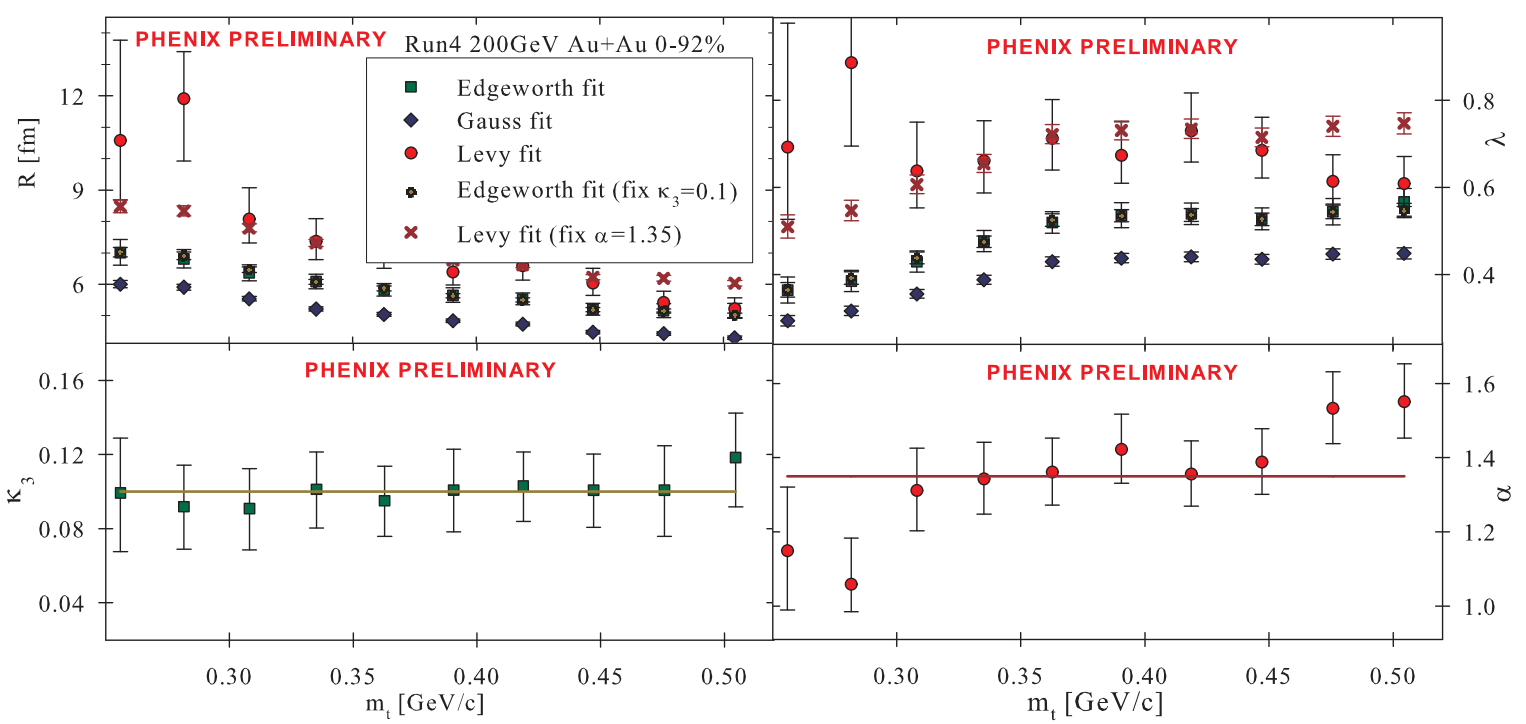

Figure 3. $\lambda\left(m_{t}\right), R\left(m_{t}\right), \alpha\left(m_{t}\right)$ and $\kappa_{3}\left(m_{t}\right)$ from Gauss, Levy and Edgeworth fits
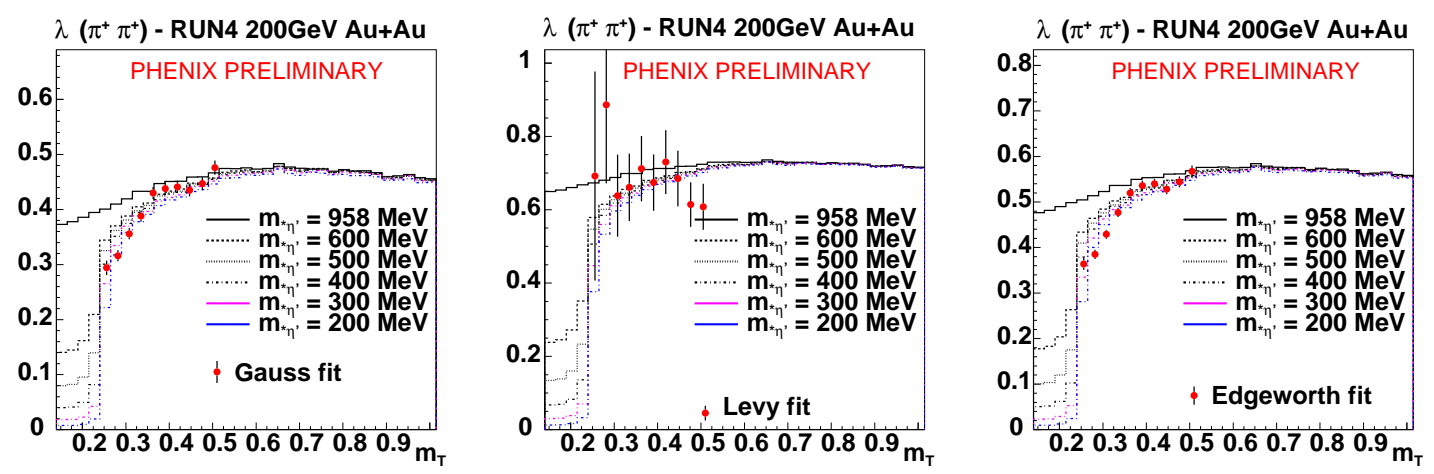

Figure 4. Measured $\lambda\left(m_{t}\right)$ compared to calculations using the model of ref. [2] with various $\eta^{\prime}$ mass values, and the Fritiof results for the composition of the long-lived resonances.

3. J. I. Kapusta, D. Kharzeev and L. D. McLerran, Phys. Rev. D 53 (1996) 5028

4. Z. Huang and X. N. Wang, Phys. Rev. D 53 (1996) 5034 arXiv:hep-ph/9507395.

5. T. Hatsuda and T. Kunihiro, Phys. Rept. 247, 221 (1994) arXiv:hep-ph/9401310.

6. T. Csörgö, B. Lörstad, J. Schmid-Sørensen and A. Ster, EPJ C 9, 275 (1999)

7. E. O. Alt, T. Csörgö, B. Lörstad and J. Schmidt-Sørensen, hep-ph/0103019

8. T. Csörgö, Heavy Ion Phys. 15 (2002) 1, hep-ph/0001233

9. H. Boggild et al. [NA44 Collaboration], Phys. Lett. B 455 (1999) 77.

10. M. Biyajima, M. Kaneyama and T. Mizoguchi, Phys. Lett. B 601, 41 (2004)

11. T. Csörgö, S. Hegyi and W. A. Zajc, Eur. Phys. J. C 36, 67 (2004)

12. S. S. Adler et al. [PHENIX Collaboration], Phys. Rev. Lett. 93, 152302 (2004) 